\title{
Energy-Efficient Transmission Strategies for Multiantenna Downlink
}

\author{
Kien-Giang Nguyen*, Oskari Tervo*, Quang-Doanh Vu*, Markku Juntti*, and Le-Nam Tran ${ }^{\dagger}$ \\ ${ }^{*}$ Centre for Wireless Communications, University of Oulu, P.O.Box 4500, FI-90014, Oulu, Finland \\ Email: \{giang.nguyen, oskari.tervo, quang.vu, markku.juntti\}@oulu.fi \\ † Department of Electronic Engineering, Maynooth University, Ireland; Email: lenam.tran@nuim.ie
}

\begin{abstract}
Energy efficiency (EE) is becoming one of the important criteria in wireless transmission design. This paper discusses the recently proposed energy-efficient transmit beamforming designs for multicell multiuser multiple-input single-output (MISO) systems, including maximizing overall network EE, sum weighted EE and fairness EE. Generally, the EE optimization problems are NP-hard nonconvex programs for which finding the globally optimal solutions is challenging. For low-complexity suboptimal approaches, there is a class of solutions conventionally developed based on parametric transformations. However, those have been revealed problematic in terms of computational complexity and convergence. To overcome these issues, novel algorithms have been recently developed based on the state-of-the-art successive convex approximation (SCA) framework. Here we sum up the basic concepts of the algorithms and provide numerical results which illustrate the solution quality compared to the existing methods.
\end{abstract}

\section{INTRODUCTION}

Fifth generation $(5 \mathrm{G})$ wireless networks visions foresee the challenges of traffic demand set by the upcoming explosive growth of wireless devices and applications [1]. To increase the achievable rates, the total energy consumption inevitably increases due to the high power feeding for the multipleantenna transmissions and involved circuit components in wireless transceivers. Thus energy efficiency (EE) has become an important criteria in cellular communications, also due to the concerns on greenhouse gas emission [2], [3].

Energy efficiency is generally defined as the ratio of the total throughput over the total power consumption of the network. It is notable that increasing the network throughput by increasing the transmit power does not always improve the achievable EE, because the power consumption also increases. Thus, finding the optimal energy-efficient operating point is essential and has become the focus in a large portion of recent works [2], [3], which investigate energy-efficient transmission strategies regarding to three main criteria, i.e., network EE (NEE), sum weighted EE (SWEE) and max-min fairness EE (maxminEE) [4]. While the first metric optimizes the EE gain of the entire network, the two other ones aim at satisfying the specific EE requirements on individual parties involved. In general, the EE maximization (EEmax) problem for each metric belongs to the class of NP-hard problems, namely fractional program for which finding a globally optimal solution is challenging. Thus, suboptimal approaches that achieve a stationary solution (i.e., a solution that satisfies the Karush-Kuhn-Tucker (KKT) conditions) have been proposed. Due to the fractional structure of the EE objective, fractional programming (FP) approaches, which are based on parametric transformation, have been typically customized for the problems [4]-[6]. However such methods result in two-layer iterative solutions [6] which often have a high computational complexity (as also discussed in Section IV-A). Moreover, analyzing the convergence of those methods is challenging and has not been addressed [7].

To overcome the issues related to the FP based solutions, novel algorithms have been recently developed to efficiently solve the maxEE problems. Those are based on the state-ofthe-art local optimization toolbox, namely successive convex approximation (SCA) algorithm. The proposed framework is a one-loop iterative procedure which finds out locally optimal solutions after a relatively small number of iterations, thus significantly reducing the complexity compared to existing FP approaches. Furthermore, the convergence of the SCA-based methods is provably guaranteed. In this paper, we make the summation of the basic concept of SCA based algorithms solving the problems of maximizing the NEE, SWEE and maxminEE. More specifically, we provide a key transformation that turns the maximization problems into representations that successfully leverage the principle of the SCA. Numerical comparison to existing methods is also provided to demonstrate the effectiveness of the proposed SCA framework.

\section{SySTEM MODEL}

\section{A. Channel and Signal Model}

We focus on a downlink transmission of multi-cell multiuser multiple-input single-output (MISO) system consisting of $B$ base stations (BS), each of which is equipped with $M$ antennas. Let $\mathcal{B}$ be the index set of BSs, i.e., $\mathcal{B}=\{1, \ldots, B\}$. Assume that each $\mathrm{BS} b \in \mathcal{B}$ communicates to $K_{b}$ singleantenna users and interferes to received signals at users of neighboring cells. For notational convenience we denote user $k$ in cell $b$ as $(b, k)$. Let $s_{b, k}$ be the data for user $(b, k)$ which is assumed to have unit energy, i.e., $\mathbb{E}\left[\left|s_{b, k}\right|^{2}\right]=1$. We also assume that linear precoding is adopted where transmit signal to user $(b, k)$ is a multiplication of the data $s_{b, k}$ and transmit beamformer $\mathbf{w}_{b, k}$. Accordingly, the received signal at user $(b, k)$ is written as 


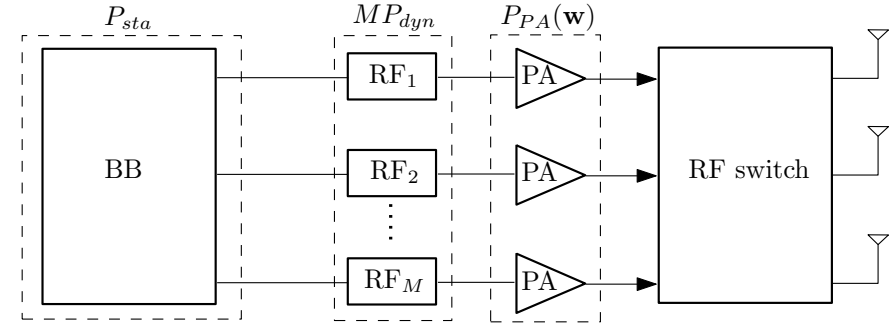

Fig. 1. Power consumption model for a BS

$$
\begin{aligned}
y_{b ; b, k}= & \mathbf{h}_{b ; b, k} \mathbf{w}_{b, k} s_{b, k}+\sum_{i=1, i \neq k}^{K_{b}} \mathbf{h}_{b ; b, k} \mathbf{w}_{b, i} s_{b, i} \\
& +\sum_{j \in \mathcal{B}, j \neq b}^{B} \sum_{i=1}^{K_{j}} \mathbf{h}_{j ; b, k} \mathbf{w}_{j, i} s_{j, i}+z_{b, k}
\end{aligned}
$$

where $\mathbf{h}_{j ; b, k} \in \mathbb{C}^{1 \times M}$ is the (row) flat fading channel vector from $\mathrm{BS} j$ to user $(b, k)$, and $z_{b, k}$ denotes the additive white Gaussian noise with the density $z_{b, k} \sim \mathcal{C N}\left(0, \sigma^{2}\right)$. In (1), the second and third terms represent the intra- and inter-cell interference. Let us denote by $I_{b, k}(\mathbf{w}) \triangleq \sum_{i=1, i \neq k}^{K_{b}}\left|\mathbf{h}_{b ; b, k} \mathbf{w}_{b, i}\right|^{2}+$ $\sum_{j \in \mathcal{B}, j \neq b}^{B} \sum_{i=1}^{K_{j}}\left|\mathbf{h}_{j ; b, k} \mathbf{w}_{j, i} s_{j, i}\right|^{2}$ the power of interference received at user $(b, k)$. Suppose that single-user detection scheme is used at all users, the received $\operatorname{SINR}$ at user $(b, k)$ is expressed as

$$
\Gamma_{b, k}(\mathbf{w})=\frac{\left|\mathbf{h}_{b ; b, k} \mathbf{w}_{b, k}\right|^{2}}{I_{b, k}(\mathbf{w})+\sigma_{b, k}^{2}}
$$

Then the data rate of user $(b, k)$ is given by $r_{b, k}\left(\mathbf{w}_{b, k}\right)=$ $\log \left(1+\Gamma_{b, k}(\mathbf{w})\right)$ and total data rate transmitting by $\mathrm{BS} b$ is $R_{b}=\sum_{b=1}^{B} r_{b, k}(\mathbf{w})$.

\section{B. Power Consumption Model}

In addition to the radiated power, energy consumption of all network components needs to be taken into consideration when evaluating the EE gain. In general the power consumed by $\mathrm{BS} b$ is illustrated as in Fig. 1 and given by

$$
P_{\text {total }, b}(\mathbf{w})=P_{\mathrm{PA}, b}(\mathbf{w})+M P_{\text {dyn }}+P_{\text {sta }}, \forall b \in \mathcal{B}
$$

In particular, $P_{\mathrm{PA}, b}(\mathbf{w})$ represents the dissipated power on the power amplifiers of the antenna chains at BS $b$. Note that $P_{\mathrm{PA}, b}(\mathbf{w})$ can adopt either linear model [8] or nonlinear model [9] which are in general the convex function of transmit beamforming $\mathbf{w}$. On the other hand, $P_{\text {dyn }}$ accounts for the dynamic power radiation of all circuit blocks in each active radio frequency chain, and $P_{\text {sta }}$ is the static power for power supply, site cooling and the baseband signal processing circuits (e.g., coding/decoding, channel estimation, synchronization, backhaul transmission, etc.). For simplicity we assume that $P_{\text {dyn }}$ and $P_{\text {sta }}$ are fixed. However, more detailed power consumption could be also applied, e.g., the power consumption for coding/decoding and backhaul can increase with the transmission rate [10].

\section{Network-CENTRIC ENERGy EFFicient Metrics}

Energy-efficient measures including the NEE, SWEE and maxminEE are discussed as follows. In general EE is defined as the ratio of achievable data rate and the total power consumption, i.e.,

$$
E E=\frac{F\left(R_{b}(\mathbf{w})\right)}{G\left(P_{\text {total }, b}(\mathbf{w})\right)}
$$

where $F\left(R_{b}(\mathbf{w})\right)$ and $G\left(P_{\text {total }, b}(\mathbf{w})\right)$ are the sum functions of data rate and power consumption, respectively. Depending on network features and design objective, the EE maximization problem is investigated under three following major criteria

Network Energy Efficiency: NEE metric is to consider the EE gain of entire network [4], [10] which follows directly the general definition of $\mathrm{EE}$ as (4), i.e.,

$$
N E E \triangleq \frac{\sum_{b=1}^{B} R_{b}(\mathbf{w})}{\sum_{b=1}^{B} P_{\text {total }, b}(\mathbf{w})}
$$

Remark that in scenarios, where cellular BSs with different features and specifications co-exist, e.g., heterogeneous network, NEE might lack relevance, since neither EE requirement for each cell nor the fairness among all parties of the network can be guaranteed.

Sum Weighted Energy Efficiency: SWEE metric is given by [6], [10]

$$
S W E E \triangleq \sum_{b=1}^{B} \omega_{b} \frac{R_{b}(\mathbf{w})}{P_{\text {total }, b}(\mathbf{w})}
$$

where $\omega_{b}$ is a parameter representing the different priority for cell $b$. The SWEE is essential in cases in which each cell has specific demand on EE. We can see that sum of multiple fraction functions in (6) is more general than the single-fraction one in (5).

Fairness Energy Efficiency: Maximizing the NEE and SWEE metrics might result in unfairness resource allocation where some links will experience low EE. If $\mathrm{EE}$ is critical for each cell, e.g., in cellular networks where BSs are not connected to fixed electricity grid, fairness EE among all parties is preferable. Towards a fairness strategy, the minimum EE among all BSs, i.e.,

$$
\min E E \triangleq \min _{b \in \mathcal{B}} \frac{R_{b}(\mathbf{w})}{P_{\text {total }, b}(\mathbf{w})}
$$

is the objective to be maximized [11].

In general, optimization problem regarding to maximizing the EE objectives (5), (6), (7) belong to the class of fractional programs. In the next section, we will briefly review conventional approaches to solve the EEmax problems, and then provide the newly developed framework which improves efficiently solution quality.

\section{SOlutions FOR ENERGY-EFFICIENT TRANSMISSIONS}

\section{A. Conventional Approaches}

To the best of our knowledge, most of existing solutions for the EEmax problems are based on customizing parameterized approaches for fractional program [4]-[6]. However, it turns 
out that the resulting algorithms are in fact a two-layer iterative procedure. More specifically, the outer loop is to determine a feasible parameter, i.e., $\eta=\frac{F\left(R_{b}(\hat{\mathbf{w}})\right)}{G\left(P_{\text {total }, b}(\hat{\mathbf{w}})\right.}$ where $\hat{\mathbf{w}}$ is a solution in the feasible set, thereby the inner loop solves the resulting parameterized problem $\max _{\mathbf{w}}\left\{F\left(R_{b}(\mathbf{w})\right)-\eta G\left(P_{\text {total }, b}(\mathbf{w})\right)\right.$ so as to derive a newly feasible $\hat{\mathbf{w}}$ corresponding to $\eta$. Moreover, this subproblem is obviously nonconvex due to the coupling of variable $\mathbf{w}$ in the SINR expression (2), and thus requires to be solved by other suboptimal optimization methods [6]. Therefore, a high number of iterations is often needed for the convergence. Furthermore, the convergence analysis of such approaches is challenging in general.

We provide below an alternative framework based on the principle of the SCA. It has been developed recently to overcome the shortcomings of the earlier solutions. Specifically, the proposed framework is fast-converging and provably converged. For the ease of exposition, we first present the SCA principle in essence before presenting its applications to the EEmax problems.

\section{B. SCA Principle}

The central idea of the SCA is to iteratively approximate the nonconvex constraints of an optimization problem by proper convex ones [12]. In particular, let us consider a general optimization program given by

$$
\min f(x) \quad \text { s.t } \quad\{g(x) \leq 0, x \in \mathcal{S}\}
$$

where $f(x)$ and $g(x)$ are convex and nonconvex functions in feasible set $\mathcal{S}$ w.r.t variable $x$, respectively. According to SCA algorithm, we need to replace $g(x)$ by a convex function $\hat{g}\left(x, y^{(n)}\right)$ at iteration $n$ where feasible point $y^{(n)} \in \mathcal{S}$ is chosen such that

(a) $\quad g(x) \leq \hat{g}\left(x, y^{(n)}\right)$

(b) $\quad g\left(x^{n}\right)=\hat{g}\left(x^{(n)}, y^{(n)}\right)$

(c) $\quad \nabla_{x} g\left(x^{(n)}\right)=\nabla_{x} \hat{g}\left(x^{(n)}, y^{(n)}\right)$

While properties (a) and (b) guarantee the monotonic convergence behavior of the SCA algorithm, combining (b) and (c) shows that the limit point obtained by the iterative procedure satisfying the KKT optimality conditions [12]. It should be kept in mind that to apply the SCA method, we need to properly find convex bounds of the nonconvex constraints which hold properties (a)-(c).

\section{Newly Developed Algorithms}

We now provide the proposed SCA framework for the energy-efficient transmit beamforming designs. Let us consider the general EE maximization problem

$$
\max \text { (4) s.t } \quad\{\mathbf{w} \in \mathcal{S}(\mathbf{w})\}
$$

where $\mathcal{S}(\mathbf{w})$ is convex feasible set of $\mathbf{w}$ which accounts for constraints on transmit power, e.g., per-BS power, per-antenna power constraints, and per-user qualify of service. Recall that (8) is noncovex due to the fractional objective and the SINR functions in $R_{b}(\mathbf{w})$. In addition, we easily check that these nonconvex parts are not the SCA applicable formulation, since they lack of convex bounds that satisfying conditions (a)-(c).
Thus, the nonconvex problem (8) needs to be translated into a more tractable representation, which lends itself to a direct application of the SCA. To this end, we use the epigraph transformation [13] by introducing new slack variables, i.e.,

$$
\begin{aligned}
\eta & \geq \frac{z^{2}}{t} \\
t & \geq G\left(P_{\text {total }, b}(\mathbf{w})\right) \\
z^{2} & \leq F\left(\log \left(1+g_{b, k}\right)\right) \\
\frac{\left|\mathbf{h}_{b, k} \mathbf{w}_{b, k}\right|^{2}}{g_{b, k}} & \geq I_{b, k}(\mathbf{w})+\sigma_{b, k}^{2}
\end{aligned}
$$

where $\eta, z, t$ and $g_{b, k}$ represent for the EE, sum data rate, total power consumption and per-user SINR, respectively. Note that $g_{b, k}$ relaxes $R_{b}(\mathbf{w})$ to concave function $\log \left(1+g_{b, k}\right)$. The problem of maximizing EE can be equivalently rewritten as

$$
\max _{\mathbf{w} \in \mathcal{S}(\mathbf{w}), \eta, z, t,\left\{g_{b, k}\right\}}\{\eta \mid(9 \mathrm{a})-(9 \mathrm{~d})\}
$$

where we can easily verify that all constraints are convex, except (9a) and (9d) holding the nonconvexity. Nevertheless, (9a) and (9d) admit the same structure, i.e., quadratic-overaffine function which is convex w.r.t. the involved variables. Thus, their upper bounds are easily derived by their first order approximations, i.e.,

$$
\begin{aligned}
\frac{z^{2}}{t} & \geq \frac{2 z^{(n)}}{t^{(n)}} z-\frac{\left(z^{(n)}\right)^{2}}{\left(t^{(n)}\right)^{2}} t \\
\frac{\left|\mathbf{h}_{b, k} \mathbf{w}_{b, k}\right|^{2}}{g_{b, k}} & \geq \frac{2 \Re\left(\mathbf{h}_{b ; b, k}^{(n)} \mathbf{w}_{b, k}\right)}{q_{b, k}^{(n)}}-\frac{\left|\mathbf{h}_{b ; b, k} \mathbf{w}_{b, k}^{n}\right|^{2} q_{b_{k}}}{\left(q_{b, k}^{n}\right)^{2}}
\end{aligned}
$$

respectively, where $\mathbf{h}_{b ; b, k}^{(n)}=\left(\mathbf{w}_{b, k}^{(n)}\right)^{H} \mathbf{h}_{b ; b, k}^{H} \mathbf{h}_{b ; b, k}$ and $(\cdot)^{(n)}$ represents feasible point at iteration $n$. More importantly, right-sides of (11) and (12) satisfy conditions (a)-(c). In the light of the SCA principle, we can replace (9a) and (9d) by (11) and (12), respectively to arrive at the approximate convex program of (10), thereby obtaining a local optimality of (10). In the following, we discuss the application of the above technique to specific EEmax problems, i.e., NEE, SWEE and maxminEE maximization.

Network Energy Efficiency: NEE maximization problem (maxNEE) is given by

$$
\max (5) \text { s.t }\{\mathbf{w} \in \mathcal{S}(\mathbf{w})\}
$$

Since (5) is similar to (4) where $F\left(R_{b}(\mathbf{w})\right)=\sum_{b=1}^{B} R_{b}(\mathbf{w})$ and $G\left(P_{\text {total }, b}(\mathbf{w})\right)=\sum_{b \in \mathcal{B}} P_{\text {total }, b}(\mathbf{w})$, we can use exactly the approach presented above to arrive at the solution. Details of the transformation, alternative transformations, and its convergence analysis can be found from [7], [10].

Sum Weighted Energy Efficiency: SWEE maximization (maxSWEE) problem is given by

$$
\max \text { (6) s.t }\{\mathbf{w} \in \mathcal{S}(\mathbf{w})\}
$$

As (6) can be viewed as generalized representation of single-fraction program (8), i.e., $\sum_{b \in \mathcal{B}} \frac{\omega_{b} F_{b}\left(R_{b}(\mathbf{w})\right)}{G_{b}\left(P_{\text {total }, b}(\mathbf{w})\right)}$ where $F_{b}\left(R_{b}(\mathbf{w})\right)=R_{b}(\mathbf{w})$ and $G_{b}\left(P_{\text {total }, b}(\mathbf{w})\right)=P_{\text {total }, b}(\mathbf{w})$, we can readily derive SCA framework solving (14) followed 
similar transformation as (9). More specifically, we introduce new slack variables for each single-fraction, i.e., for $b \in \mathcal{B}$,

$$
\eta_{b} \geq \frac{z_{b}^{2}}{t_{b}} ; t_{b} \geq P_{\text {total }, b}(\mathbf{w}) ; z_{b}^{2} \leq \sum_{k=1}^{K_{b}} \log \left(1+g_{b, k}\right)
$$

and (14) is rewritten as

$$
\max _{\mathbf{w} \in \mathcal{S}(\mathbf{w}),\left\{\eta_{b}\right\},\left\{z_{b}\right\},\left\{t_{b}\right\},\left\{g_{b, k}\right\}} \sum_{b=1}^{B} \omega_{b} \eta_{b} \text { s.t } \quad\{(9 \mathrm{~d}),(15)\}
$$

In fact, (16) admits a similar structure to (10), but has larger problem size. In the same manner, the SCA based algorithm solving (16) can be followed the one solving (10). More details of the proposed method solving (16) can be found in [10] and an alternative formulation in [14].

Max-Min Fairness Energy Efficiency: The problem of maxminEE is given by

$$
\max \text { (7) s.t }\{\mathbf{w} \in \mathcal{S}(\mathbf{w})\}
$$

Note that (7) can be relaxed to a similar problem as (14) by adding a slack variable, i.e.,

$$
\max _{\mathbf{w} \in \mathcal{S}(\mathbf{w})} \eta \text { s.t }\left\{\frac{R_{b}(\mathbf{w})}{P_{\text {total }, b}(\mathbf{w})} \geq \eta\right\}
$$

where $\eta$ now represents for the minimum EE among all BSs. Thus we can utilize the transformation for $\frac{R_{b}(\mathbf{w})}{P_{\text {total }},(\mathbf{w})}$ as in (15) to arrive at SCA applicable formulation. We refer to [11] for complete convergence analysis and related discussion of method solving (17).

\section{Distributed Implementation}

We remark that the algorithms mentioned above are designed in the centralized fashion under assumption that each BS perfectly knows all channel state information in the network, as well as all computation is done centrally. However, this may be not practical appealing since they come at expense of building central nodes collecting associated information to serve the computation. From the practical implementation perspective, distributed solutions are more attractive. Note that the FP approaches are not suitable for decentralized implementation, since updating the parameterized value requires a centralized calculation with all per-BS EEs. In contrast, the SCA based algorithm can be easily extended for a decentralized mechanism. In fact, distributed algorithm based on combination of the SCA and alternating direction method of multiplier methods (ADMM) have been proposed in [14], [15] and closed-form implementations based on the KKT optimality conditions in [10]

\section{NUMERICAL RESULT}

We now demonstrate the effectiveness of the proposed SCA based algorithms. The simulation parameters are listed in Table I and specific ones are given in the caption of the corresponding figures. For comparisons, we additionally provide the performances of FP methods [6].

Fig. 2 compares the convergence speed of the algorithms based on SCA framework and those of FP. In particular, snapshot of the gap between the objective value at the $n$th
Table I

SIMULATION PARAMETERS

\begin{tabular}{c|c}
\hline PARAMETERS & VALUE \\
\hline \hline Path loss and shadowing & $38 \log _{10}(d[\mathrm{~m}])+34.5+\mathcal{N}(0,8)$ \\
Inter-BS distance & $D=1 \mathrm{~km}$ \\
Dynamic power consumption $P_{\mathrm{dyn}}$ & $30 \mathrm{dBm}$ \\
Static power consumption $P_{\mathrm{dyn}}$ & $33 \mathrm{dBm}$ \\
Power amplifier efficiency $\epsilon$ & 0.35 \\
Number of BSs $B$ & 3 \\
Number of users per cell $K_{b}$ & 2 \\
Number of Tx antennas $N$ & 4 \\
Signal bandwidth $W$ & $10 \mathrm{kHz}$ \\
Power spectral density of noise & $-174 \mathrm{dBm} / \mathrm{Hz}$ \\
\hline
\end{tabular}

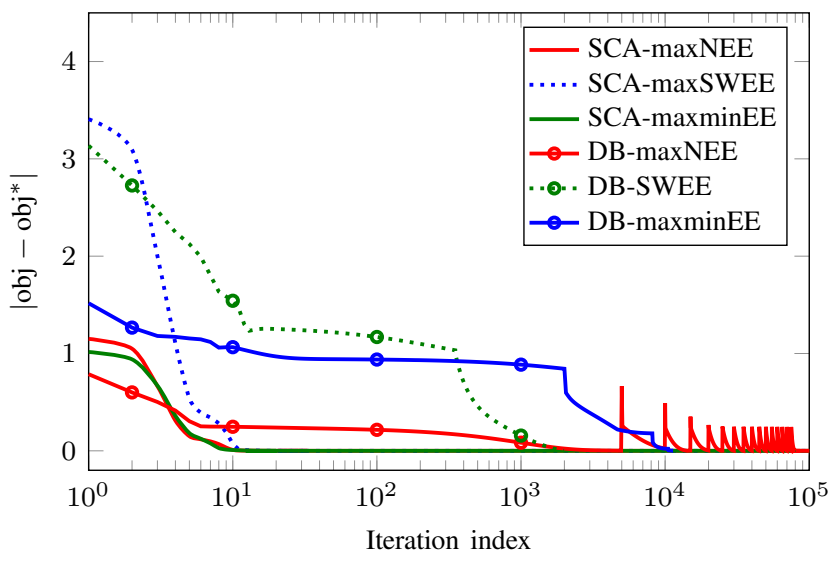

Fig. 2. Convergence rate with transmit power budget $40 \mathrm{dBm}$

iteration and the one at convergence, i.e., $\left|\mathrm{obj}_{-}-\mathrm{obj}^{*}\right|$ for one channelization is illustrated. As can be seen, the SCA based methods monotonically converge after ten iterations. On the other hand, the other ones require hundreds to thousands of iterations to converge if each subproblem is solved with high accuracy. This implies that the proposed SCA framework significantly reduces the complexity. In addition, FP framework does not always guarantee the monotonic convergence.

In Fig. 3 we show the achievable EEs versus the power budget by different energy efficiency criteria in terms of the NEE, sum EE and min EE. Users of BS 2 are set to have worse channel conditions to their serving BS than those of BS 1, but better than those of BS 3. As can be seen, maxNEE obtains better NEE performance due to the optimization mechanism regarding to NEE objective. For maxSWEE scheme, assigning different weighted priorities, e.g., $\boldsymbol{\omega}_{1}$ and $\boldsymbol{\omega}_{2}$, can change significantly the achieved performances. Note that $\omega_{1}$ implies higher priority to BS 1 whose users have good channel condition to the serving BS, while $\boldsymbol{\omega}_{2}$ gives high-demand EE to BS 3 which has worse channels to its users. Thus, it is easily understood that using weighted vector $\boldsymbol{\omega}_{1}$ results in higher achieved NEE and sum EE compared to using $\boldsymbol{\omega}_{2}$ as in Figs. 3 (a) and $3\left(\right.$ b). However, assigning $\omega_{2}$ improves remarkably the achieved min EE than using $\boldsymbol{\omega}_{1}$ (see Fig. 3(c)). For maxminEE scheme, it outperforms the other two schemes in terms of min EE as shown in Fig. 3(c), since the min EE is objective to be maximized.

In addition, maxminEE is able to guarantee the fairness among all parties as shown in Fig. 4, which plots the fairness 


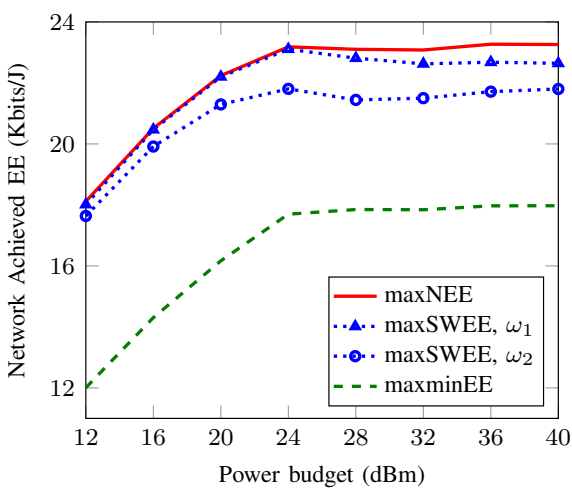

(a) Network EE

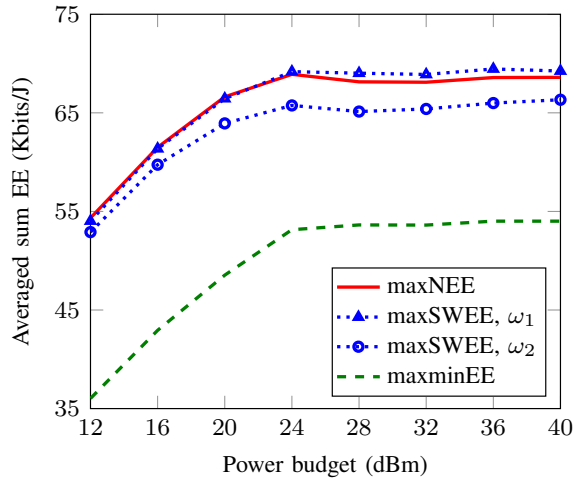

(b) Sum EE

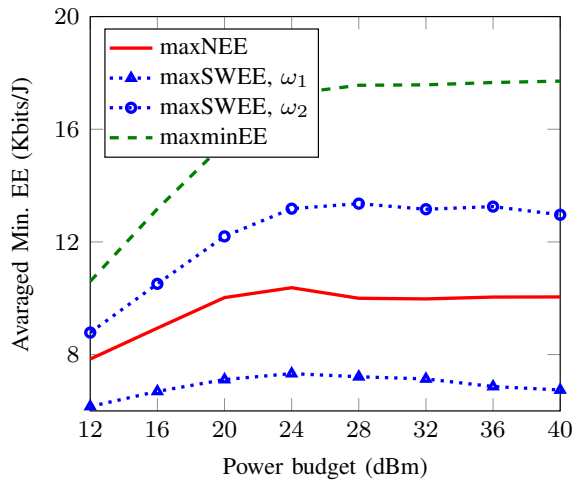

(c) Min EE

Fig. 3. Averaged EE versus transmit power budget. For maxSWEE, we use two sets of priority parameters, i.e., $\boldsymbol{\omega}_{1}=[0.7,0.5,0.3]$ and $\boldsymbol{\omega}_{2}=[0.3,0.5,0.7]$.

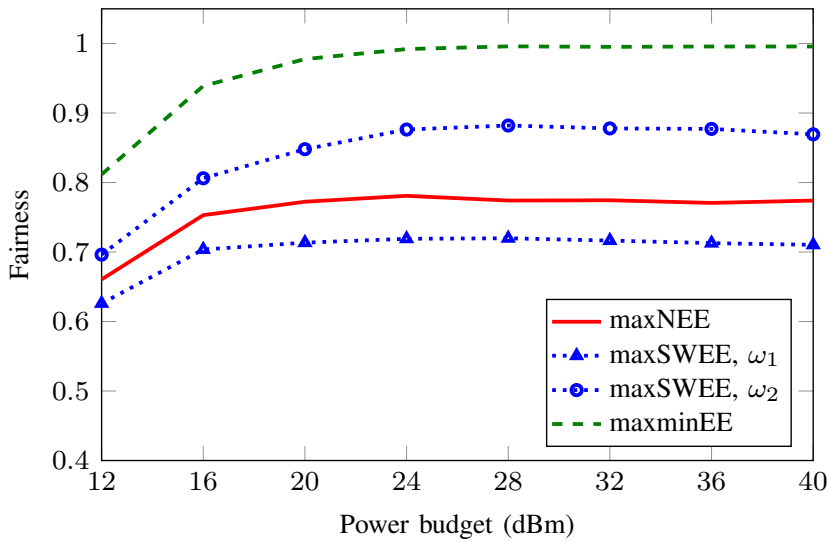

Fig. 4. Fairness versus power budget

metric [16, Eq. (1)] achieved by different EE strategies. On the other hand, we can see that maxSWEE scheme can adjust the EE fairness among involved parties by setting priority in the network. On the contrary, the fairness cannot be improved by the maxNEE strategy as it depends on the entire network configuration than the individual elements.

\section{CONCLUSION}

We have provided a summary and performance comparison of low-complexity algorithms for the problems of EE optimization in multicell multiuser MISO downlink, under three energy efficiency metrics. The proposed algorithms are based on the principle of the SCA methods in which the nonconvex problems corresponding to fractional program are handled efficiently. This has been shown by the numerical results where SCA based algorithms outperform the existing FP ones in terms of convergence speed. This paper can be viewed as a guideline for the application of the SCA in solving the energyefficient beamforming designs in particular, and the nonconvex problems in wireless communications in general.

\section{REFERENCES}

[1] Qualcomm, "The 1000x data challenge," tech. rep. [Online]. Available: http://www.qualcomm.com/1000x.

[2] D. Feng, C. Jiang, G. Lim, J. Cimini, L. J., G. Feng, and G. Li, "A survey of energy-efficient wireless communication," IEEE Commun. Surveys Tuts., vol. 15, no. 1, pp. 167-178, Feb. 2013.
[3] G. Y. Li, Z. Xu, C. Xiong, C. Yang, S. Zhang, Y. Chen, and S. Xu, "Energy-efficient wireless communications: tutorial, survey, and open issues," IEEE Wireless Commun., vol. 18, no. 6, pp. 28-35, 2011.

[4] A. Zappone and E. Jorswieck, "Energy efficiency in wireless networks via fractional programming theory," Foundations and Trends in Communications and Information Theory, vol. 11, no. 3-4, pp. 185-396, 2015.

[5] D. W. K. Ng, E. S. Lo, and R. Schober, "Energy-efficient resource allocation in multi-cell OFDMA systems with limited backhaul capacity," IEEE Trans. Wireless Commun., vol. 11, no. 10, pp. 3618-3631, Oct. 2012.

[6] S. He, Y. Huang, L. Yang, and B. Ottersten, "Coordinated multicell multiuser precoding for maximizing weighted sum energy efficiency," IEEE Trans. Signal Process., vol. 62, no. 3, pp. 1005-1017, Feb. 2014.

[7] O. Tervo, L.-N. Tran, and M. Juntti, "Optimal energy-efficient transmit beamforming for multi-user MISO downlink," IEEE Trans. Signal Process., vol. 63, no. 20, pp. 5574 - 5588, Oct. 2015.

[8] O. Arnold, F. Richter, G. Fettweis, and O. Blume, "Power consumption modeling of different base station types in heterogeneous cellular networks," in Proc.19th Future Network \& Mobile Summit (ICT Summit'10), Florence, Italy, Jun. 2010, pp. 1-8.

[9] D. Persson, T. Eriksson, and E. G. Larsson, "Amplifier-aware multipleinput multiple-output power allocation," IEEE Commun. Lett., vol. 17 no. 6, pp. 1112-1115, June 2013.

[10] O. Tervo, A. Tölli, M. Juntti, and L.-N. Tran, "Energy-efficient beam coordination strategies with rate dependent processing power," arXiv preprint arXiv:1610.02307, 2016.

[11] K.-G. Nguyen, L.-N. Tran, O. Tervo, Q.-D. Vu, and M. Juntti, "Achieving energy efficiency fairness in multicell multiuser MISO downlink," IEEE Commun. Lett., vol. 19, no. 8, pp. 1426 - 1429, Aug. 2015.

[12] B. R. Marks and G. P. Wright, "A general inner approximation algorithm for nonconvex mathematical programs," Operations Research, vol. 26 , no. 4, pp. 681-683, Jul.-Aug. 1978.

[13] S. Boyd and L. Vandenberghe, Convex optimization. Cambridge university press, 2004.

[14] O. Tervo, L. N. Tran, and M. Juntti, "Decentralized coordinated beamforming for weighted sum energy efficiency maximization in multicell miso downlink," in 2015 IEEE Global Conference on Signal and Information Processing (GlobalSIP), Dec 2015, pp. 1387-1391.

[15] K. G. Nguyen, L. N. Tran, Q. D. Vu, and M. Juntti, "Distributed energy efficiency fairness optimization by admm in multicell MISO downlink," May 2016, pp. 1-6.

[16] R. Jain, D.-M. Chiu, and W. R. Hawe, A quantitative measure of fairness and discrimination for resource allocation in shared computer system. Eastern Research Laboratory, Digital Equipment Corporation Hudson, MA, 1984, vol. 38 . 